\title{
Identifying Main Factors of Knowledge Transfer in Humanities and Social Science in an Individual Perspective: A Structure Equation Modeling
}

\author{
Xiaofei Jin ${ }^{1, a}$, Huiying Zhang ${ }^{2, b}$ \\ ${ }^{12}$ College of Management \& Economics, Tianjin University, Tianjin, 300072, China \\ a bemail: hyzhang@tju.edu.cn
}

Keywords: humanities and social science; knowledge transfer; structure equation modeling

\begin{abstract}
In this paper, humanities and social science scholars were regards as study object, and the influential elements of transform of humanities and social science achievements were fixed on research motivation, perceived research environment, research information and dissemination approach. Some explicit relations among the transform effect and each variable were obtained by analysis with structure equation modeling. Finally, the key factors were gained.
\end{abstract}

\section{Introduction}

The extent to which humanities and social science research advanced has already become the symbol of comprehensive national strength. It plays a central and critical role in economy, science and technology. The synthesis of prior studies has revealed an overlook of the knowledge transfer in humanities and social science research. Social science research results are shown as publication, scholarly journal, report or books which are applied by policy-makers, practitioners and social science scholars to formulate policies, make decisions and support one's study [1] [7].

In recent 10 years, knowledge transfer has been regard as a theoretical issue, yet the state of analysis remains relatively scattered and non-construction. Our study focus on the earlier stage of knowledge transfer and regard research results as information to be sent, which indicates that for knowledge transfer to occur effectively, the research should be high-quality and spread by exact mode of transmission. Further more, we derive factors including internal individual and external environment perspectives from the prior researches, and then examine the possible role of these factors. This study not only aims to contribute to the empirical analysis of knowledge transfer in humanities and social science but also has implications for social science researchers intending to pursue academic engagement and for policy-makers seeking to promote knowledge transfer effect.

\section{Literature review and hypothesis}

Prior study develops a conceptual framework model to describe how technology transfer took place, which involved transferor (the owner or holder of the knowledge), transferee (recipient and the beneficiary), technology, mechanism of transfer and external environment [6]. This paper concentrates on the elements related to transferor in an individual perspective, as research motivation in psychological perspectives, perceived research environment, research information and dissemination approach.

Individual factors play a key role in contributing to knowledge transform. The present study adopts a broader and psychologically richer notion of motivation to incorporate its extrinsic as well as intrinsic aspects. It employs the three concepts of 'gold' (financial rewards like salary and funding), 'ribbon' (reputation and career rewards like the recognition and prestige attained from academic peers) and 'puzzle' (intrinsic satisfaction of advancing new knowledge) [1]. In this paper, they are defined as vegetative motivation, social motivation and self-fulfillment motivation. Motivation is the outcome of interaction between external regulatory processes and individuals' internal psychological needs for autonomy and self-determination [2].

H1: Research motivation has a positive impact on the effect of knowledge transfer. 
H2: Research motivation has a positive impact on the utilization of dissemination approach.

Previous studies indicate that it is important to consider social capital in knowledge transfer activity. Social capital is described as one's social ties to other individuals, groups, or organizations [4]. Social networks support researchers to contribute new knowledge to society [5]. External environment provide transferors with a set of conditions to nurture the new potential idea and reference to support. From an individual perspective, transferors are influenced by their perception of research environment which are external scientific research conditions of acquiring social capital. It includes four concepts as management environment (the service provided by research management to ensure the accomplishment of scientific research), intellectual atmosphere (the climate in which researcher exchanges ideas and communicates with other academic peers), social-support environment (the efforts to support the scientific research by government or enterprises) and hardware (various material conditions such as funding and research resource).

H3: A perception of research environment has a positive impact on the effect of knowledge transfer.

H4: A perception of research environment has a positive impact on the utilization of dissemination approach.

Knowledge transfer research has already studied the individual's current human capital which contains an individual's knowledge and skills acquired from the experiences increasing researchers' productivity at work [4]. Transferors' ability to transform knowledge to society is closely related to human capital endowments. In general, social scientific research begins with the identification of the social reality issue, the needs of practitioners and decision makers become the weather vane of scientific research in social science [3]. As a result, researchers are concerned with the social and economic problems based on prior relevant studies, so the comprehensive and effect research information is necessary. It includes three concepts as topic option information (determine the research orientation and derive from a dynamic social phenomenon), reference information (the academic information from previous studies) and researchers' ability of getting information.

$\mathrm{H} 5$ : the extent of grasping research information has a positive impact on the effect of knowledge transfer.

H6: the extent of grasping research information has a positive impact on the utilization of dissemination approach.

Previous researches suggest that the transformation from research knowledge to productivity cannot be autonomous and a step should be added by developing dissemination mechanisms to identify useful knowledge and transfer it to potential users [3]. It treats dissemination as a facilitator in knowledge transfer. In this case, transferee decides the dissemination approach since transferee is the recipient who benefits from knowledge transfer activity [6]. When the potential transferees focus on the research results, the knowledge transfer will take place. Dissemination approach includes three concepts as traditional print medium (publishing books, report and journal article to popularize research results), meeting (transferors participate in symposium or seminar to communicate with policy-maker or other scholars) and digital media (television or internet).

H7: The utilization of dissemination approach has a positive impact on the effect of knowledge transfer.

The results of humanities and social science study are mainly applied to social life, besides they exert a direct effect on social activities. Thus, we defined effect of knowledge transfer as the degree of the interaction between research result and social activity, which includes the extent of knowledge transfer and the intensity of knowledge transfer.

\section{Research methodology and Results}

The paper draws on the data derived from the questionnaire survey for empirical study. The sample population randomly selected from the social science researchers in 10 main research institutions in Tianjin. The questionnaire was send to about 200 academics, acquired 195 questionnaires, giving a $97.5 \%$ response rate. We subsequently yielded 14 responses as most of them missed index or had the same option, and effective return ratio is $92.8 \%$. 
The distribution of survey samples indicates that a large range of samples are involved in this survey which could be representative of all the research objects. This paper adapts reliability analysis to examine the reliability of questionnaire and the reliability coefficient is 0.727 . The consistency of items suggests the development of questionnaire is proper and scientific, thus the results of survey is reliable. In this study, a SEM program LISREL8.5 was used to test the data from measure model in the path diagram of initial model. The results suggest that the normed chi-square ( $x^{2} / \mathrm{df}$ ) is slightly greater than 2 . The value of RMSEA is between 0.08 and 0.10 as well as the value of GFI, AGFI, NFI, NNFI and CFI exceed 0.8. Thus, it is acceptable to develop the structure models based on this measurement models. So we dealt with the initial model to gain the path diagram of initial model and the fit indices of initial model. The results show that GFI, AGFI, NFI, NNFI, CFI is not acceptable in initial model, the modification of model is necessary. The results of LISREL program provide the modification index. The final model (Fig.1) and the fit indices are acquired. Obviously, the fit indices of final model are acceptable compared them in the initial model. Therefore we can assume that the final model in our research has certain explanatory.

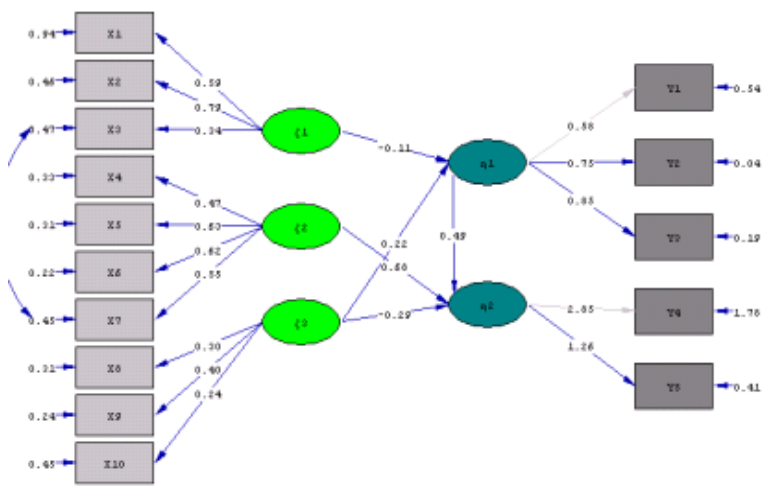

Fig.1. The path diagram of final mode

Table I The parameter estimation of patent variable to observed variable and patent variable in final model

\begin{tabular}{|l|l|l|l|}
\hline & Parameter Estimated value & Standard error & t-value \\
\hline$\lambda_{\mathrm{x} 11}$ & 0.59 & 0.098 & $6.05^{*}$ \\
\hline$\lambda_{\mathrm{x} 21}$ & 0.79 & 0.096 & $8.23^{*}$ \\
\hline$\lambda_{\mathrm{x} 31}$ & 0.34 & 0.066 & $5.24^{*}$ \\
\hline$\lambda_{\mathrm{x} 42}$ & 0.47 & 0.053 & $9.01^{*}$ \\
\hline$\lambda_{\mathrm{x} 22}$ & 0.63 & 0.055 & $11.55^{*}$ \\
\hline$\lambda_{\mathrm{x} 62}$ & 0.62 & 0.051 & $12.20^{*}$ \\
\hline$\lambda_{\mathrm{x} 72}$ & 0.55 & 0.061 & $9.06^{*}$ \\
\hline$\lambda_{\mathrm{x} 83}$ & 0.30 & 0.052 & $5.85^{*}$ \\
\hline$\lambda_{\mathrm{x} 93}$ & 0.40 & 0.050 & $7.98^{*}$ \\
\hline$\lambda_{\mathrm{x} 10,3}$ & 0.24 & 0.054 & $4.40^{*}$ \\
\hline$\lambda_{\mathrm{y} 11}$ & 0.58 & & \\
\hline$\lambda_{\mathrm{y} 21}$ & 0.75 & 0.075 & $9.95^{*}$ \\
\hline$\lambda_{\mathrm{y} 32}$ & 0.85 & 0.085 & $10.07^{*}$ \\
\hline$\lambda_{\mathrm{y} 42}$ & 2.85 & & \\
\hline$\lambda_{\mathrm{y} 52}$ & 1.26 & 0.13 & $9.88^{*}$ \\
\hline$\gamma_{11}$ & -0.11 & 0.100 & -1.14 \\
\hline$\gamma_{22}$ & 0.68 & 0.29 & $2.37^{*}$ \\
\hline$\gamma_{13}$ & 0.22 & 0.10 & $2.15^{*}$ \\
\hline$\gamma_{23}$ & -0.29 & 0.30 & -0.97 \\
\hline$\beta_{21}$ & 0.49 & 0.079 & $6.26^{*}$ \\
\hline$* \mathrm{P}<0.05$ & & & \\
\hline
\end{tabular}

From Table I, it is apparently that t-value of all the observed variables are greater than 1.96, which reveals that the observed variables reflect the patent variables positively and we can conclude that the path coefficients of research motivation to dissemination approach and knowledge transfer effect are less for 1.96, which means the effect of motivation is not positive. 
The results of our research reveal that a positive perception of research climate has a positive impact on the effect of knowledge transfer. The first major findings show that the research climate can be a facilitator of knowledge transfer. The extent to which researchers satisfied with research environment influence the effect of knowledge transfer. The academic exchange is described as researchers interact and integrate new ideas, new method and the present academic front with their professional peers. This process is associated with research management environment. The intellectual exchange can be established by a scientific and reasonable research management mechanism which provides more opportunities for researchers to exchange their new knowledge and methods. In addition, the decision-maker should ensure the establishment of academic exchange environment to encourage researcher to transfer knowledge and structure people oriented research development concept. In addition,

The application of dissemination approach has a positive impact on the effect of knowledge transfer. The second major findings suggest that meeting refers to a face-to-face dissemination approach. A pervious study from Harvard University and Columbia University has indicated that the face-to-face meeting can attract more than 38\% attention from transferee so that it is the most efficient and effective way to deliver information. However, meeting is high-cost and limited by the great distance or other elements. As transferor send information to large-scale group or transferee in distant place, another medium is available. Within the traditional model, publication is the main currency in the exchange relationship [1]. Compared with traditional print medium, we can find that the digital media is superior as it provides service with the characteristics of convenience, coverage, immediacy, rich-content and having various retrieval methods. With the development of science and technology, promoting the application of digital media in transferors is beneficial to spread the scientific research results.

The research information occupied by transferor has a positive impact on the choice of dissemination approach. Therefore, it indirectly influences the effect of knowledge transfer. Scientific research begins with selecting topic which plays a central role in completing the study. Information of research topic has a direct effect on the target and content of analysis process, which determines the tendency of research. Further more, it is a symbol for a level of research quality as well, and then performs a key role within knowledge transform. Reference information is regard as an object which humanities and social science researcher deal with and is a crucial ingredient for the development of research process. High-quality research result generally developed their arguments and thesis on the basis of former studies, which is supported by the reference information of high academic values. In order to achieve the transformation of knowledge, transferors should grasp the multiple channels to get a large amount of valuable information.

To find out the causes, we examined the correlation between exogenous observed variables of research motivation, dissemination approach and effect of knowledge transfer. The result illustrates that part of variables are positively linked to dissemination approach, while part of them have negative influence on it. Hence, this could hinder the correlation between research motivation and dissemination approach. This finding suggests that motivation is a complex, multilevel and multidimensional phenomenon. As for, we simply focus on the demands of researchers which could not reflect the interaction between motivation and effect of knowledge transfer. Clearly, the follow-up studies should develop on the basis of other aspects of motivation theory in psychology to test direct links between motivation and effect of knowledge transfer.

\section{Acknowledgment}

The research was funded by the Key Subject of the Twelfth Five-year Plan in Education Science in Tianjin (CE1006), the Planned Program of Philosophy and Social Science Research in Tianjin (TJJXTW10-30). Other support was provided by the Major Project in Social Science of Education Committee in Tianjin in 2011 (2011ZD010). 


\section{References}

[1] Alice Lam. What motivates academic scientists to engage in research commercialization: 'Gold' , 'ribbon' or 'puzzle' ? [J].Research policy, 2011, 40: 1354-1368.

[2] Gagne, M., Deci, E.L.. Self-determination theory and work motivation [J].Journal of Organizational Behavior, 2005, 26 (4): 331 - 362.

[3] Réjean Landry, Nabil Amara, Moktar Lamari. Utilization of social science research knowledge in Canada[J].Research policy, 2001, 30:333-349.

[4] Maximilian Goethner, Martin Obschonka, Rainer K. Silbereisen, Uwe Cantner. Scientists' transition to academic entrepreneurship: Economic and psychological determinants [J].Journal of Economic Psychology, 2012, 33: 628-641.

[5] Bozeman, B., \& Mangematin, V.. Editor's introduction: Building and deploying scientific and technical human capital [J].Research Policy,2004, 33(4):565 - 568.

[6] Navid Khabiri, Sadegh Rast, Aslan Amat Senin. Identifying Main Influential Elements in Technology Transfer Process: A Conceptual Model [J]. Procedia social and behavioral sciences, 2012, 40:417-423.

[7] Elena castro, Martinez. The knowledge transfer in humanities and social science: the informal relationship in Public research institutions [J].Social science research abroad, 2012, 6:59-67. 